\title{
CAPTURING THE ENVIRONMENT: using photogrammetry to register the built environment for simulation
}

\author{
Olavo Avalone Neto \\ Universidade Federal de Santa Maria | Brazil | olavo.neto@ufsm.br
}

Marianne Costa Avalone

Universidade Federal de Santa Maria | Brazil | arq.marianne.costa@gmail.com

\begin{abstract}
This study tested two forms of data gathering, three different methods of data registration, and two of modeling for the creation of 3D models of heritage landmarks. The applications on elements of three different scales were tested, a Cathedral, a Monument, and an Art Panel. The open-source Meshroom resulted in the best model in measures of mesh detail, reconstruction capability, and mesh refinement, regardless of the data acquisition method. Results may aid researchers and designers in choosing a workflow that suits their needs developing the best model possible, according to the tools they have at their disposal.
\end{abstract}

Keywords: Photogrammetry; Mesh modeling; Reality capture; Cultural heritage; 3D models.

\section{INTRODUCTION}

The measurement and registry of the built environment are continuously improving, allowing urban planners, architects, and urban designers to design using better and more accurate models and for the present state of heritage sites to be accurately recorded (Peña-Villasenín, Gil-Docampo, \& Ortiz-Sanz, 2020).

Today it is relatively common the use of structure from motion (SfM) photogrammetric methods to register the built environment at different building states (pre-design state, as-built, cultural heritage) and use these registries for design presentation, research, and for the design process itself (Achille et al., 2015; Murphy, McGovern, \& Pavia, 2013; Sužiedelytè-Visockienè, Bagdžiūnaitè, Malys, \& Maliene, 2015). However, the procedures for data gathering, processing, and producing a digital model for design applications are still fuzzy, with different workflows resulting in models of varying quality.

This paper explains the workflow adopted for creating LOD3 mesh models of three heritage landmarks of the city of Cachoeira do Sul, in southern Brazil. It describes the different data collection, registration, and modeling methods adopted and suggests a workflow based on project scale, types of software license available, and required model precision.

\section{D RECONSTRUCTION OF THE ENVIRONMENT}

A strategic review by the International Society for Photogrammetry and Remote Sensing (ISPRS) has revealed that generating $3 \mathrm{D}$ geometry from photographs through automated or semi-automated data has been improving in many fronts, such as the popularization of UAV, switch from single to integrated sensors, active sensors, improvements in methods for the orientation of image and range sensors and object reconstruction, replacement of separate by combined processing of images and point clouds, developments in multi-scale, ndimensional data modeling, and shift from mapping to monitoring and dynamic geospatial services (Heipke, Madden, Li, \& Dowman, 2016).

According to Peña-Villasenín et al. (2020), over the past few years, there have been significant improvements in the field, primarily due to improvements in data registration software, such as automation of image feature matching, automatic identification of the matching points in stereo pairs of photographs, automation of the calibration process, use of CMVS and PMVS2 algorithms for automatic 3D modeling of complex geometries, and continuous advancements in computer capabilities. Diminishing costs and popularization of drones, which allows for high precision data capture, and the ubiquitous availability of GPS tagged cameras through smartphones, have also contributed. El-Hakim (2001) also points out that several companies now provide a wide range of software to process photographic data for the $3 \mathrm{D}$ reconstruction of the environment. Improvements in the field follow three main fronts: detail capture, automation, and geometric accuracy.

CityGML standards classify 3D models into five different levels of detail, as defined by LODO (2,5D building footprints and roof edge polygons); LOD1 (extruded footprints or prismatic models); LOD2 (simplified models with differentiated roof structures and semantically enriched boundary surfaces); LOD3(detailed architectural models with openings such as windows and doors) and LOD4, that are models with similarly detailed indoor geometries of buildings (Boeters, Arroyo Ohori, Biljecki, \& Zlatanova, 2015; Gröger, Kolbe, Nagel, \& Häfele, 2012).

The 3D reconstruction method adopted for this study utilizes software that provides geometric accuracy (Peña- 

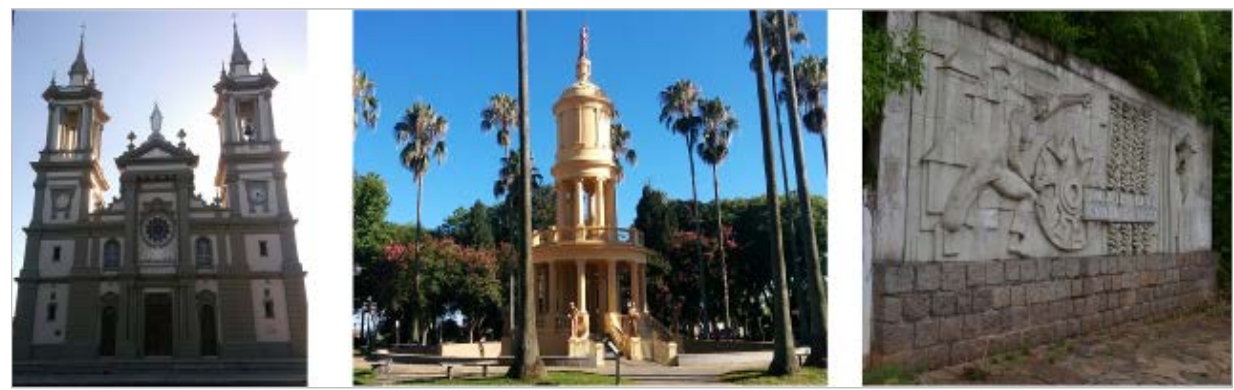

Figure 1: The three objects of interest studied.

Villasenín et al., 2020) and automation for the generation of LOD3 models.

\section{METHODOLOGY}

For the modeling goals of this study, we selected three heritage landmarks in the city of Cachoeira do Sul, in Southern Brazil: a Cathedral, a Monument, and an Art Panel (Fig. 1).

We sought to create 3D mesh models of these landmarks from a series of photographs in a process commonly known as photogrammetry. The workflow which encompasses this process includes a sequence of three activities: data collection, data registration, and modeling (El-Hakim, 2001). To analyze the suitable tools to develop the sequence, while considering the different project scales, the software license types, and model precision, we adopted two different ways of data collection, three types of data registration, and two types of modeling. The methods adopted in each activity will be thoroughly described in the following lines.

\section{DATA COLLECTION}

The Cathedral and the Monument's height required a drone flight for data collection. A free flight using a GPS tagged DJI Phanton 4 Pro captured 190 pictures of the Cathedral and 174 pictures of the Monument, from various heights and viewpoints. Although an automated flight with pre-configured picture overlap was desirable, it was no viable due to surrounding obstacles, so a manual flight was adopted. Figure 2 illustrates the camera positions related to the Monument in the point cloud

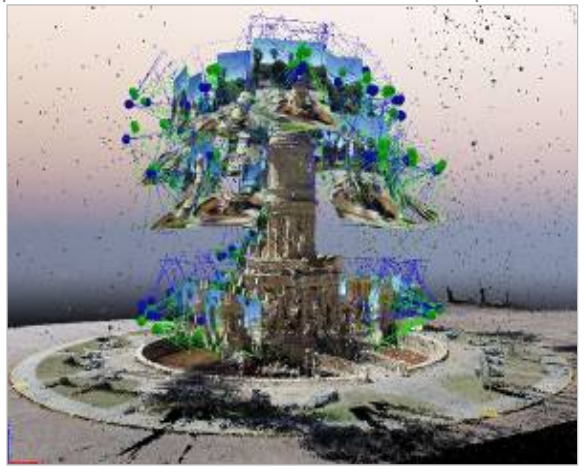

Figure 1: Monuments' Point Cloud in Pix4D, with camera positions. generated by the software Pix4Dmapper. For the Panel, we used a DSLR camera without GPS and took pictures from several standpoints and two different heights using a selfie-stick. A total of 345 pictures of the Art Panel were captured. The vantage points can be seen in the point cloud generated by the software Meshroom in Figure 3.

\section{DATA REGISTRATION}

The pictures collected were processed through three different software: a proprietary software (Pix4Dmapper), a proprietary software made available with restrictions (Autodesk ReCap Photo), and open-sourced software (Meshroom). Data registration and the resulting model

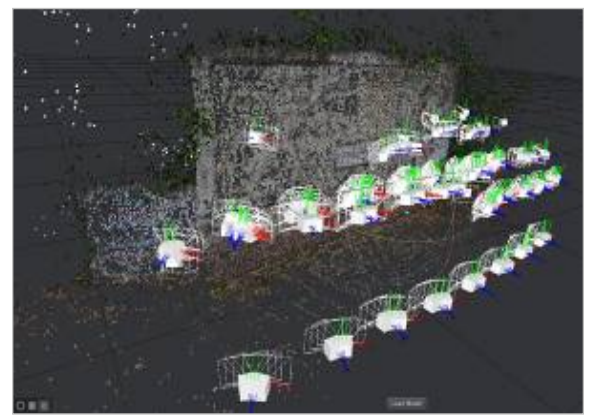

Figure 3: Art Panel's Point Cloud in Meshroom, with camera positions.

quality were conditioned by the software capabilities and limitations considering free access. For benchmarking purposes, in this paper, we adopted each software's default settings for data registration. Though each software had its particularities, which will be described in the results, we preferred to keep them untouched to prevent further distortions to the analysis.

The first software tested was Pix4Dmapper, a powerful software developed by Pix4D, specifically for research with photogrammetry and algorithmic processing of images captured with drones. It is proprietary software and offers a 15-day trial available through an e-mail registration. With default settings, the $3 \mathrm{D}$ textured mesh was set with a medium resolution, although the software allows the generation of a more detailed model with a high definition option. From the initial processing, Pix4Dmapper creates a simplified point cloud. Then, it allows users to fine-tune the model, including anchor points, correcting misinterpretations of vertices, classifying pixel areas in specific photos, and creating masks, 


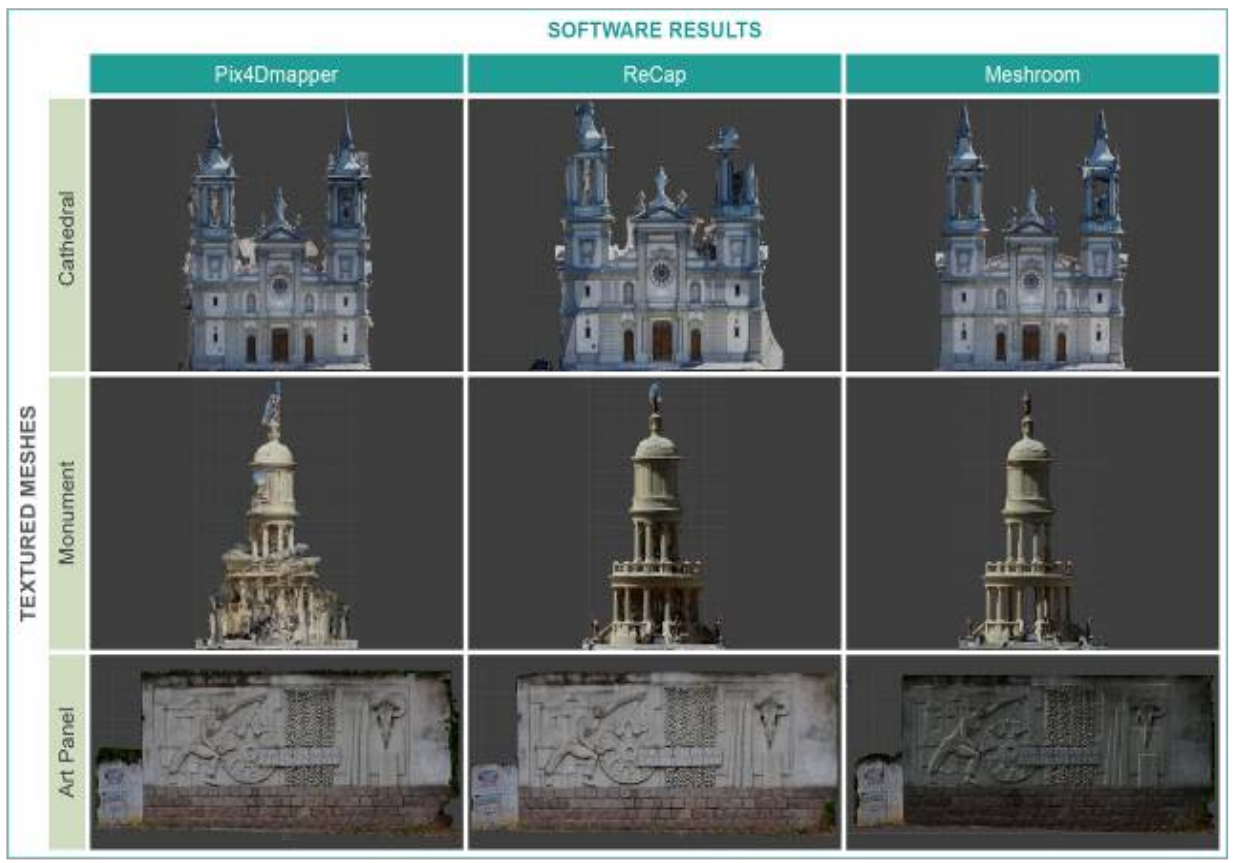

Figure 4: Textured model results in different software.

allowing for non-destructive edits that may be refined until a high-quality model can be generated from a densified point cloud. Due to its 15-day trial limitation, it could be challenging for first-time users to take full advantage of its functionality without purchasing a license.

Next, we tested the software Autodesk ReCap Photo, which is also proprietary, but it is free for non-commercial use. That makes it accessible for students, teachers, and researchers, but it brings some limitations. The main limitation is the number of pictures allowed per model, limited to a hundred. The software uses cloud processing, providing a model ready for fine-tuning and download. It brings a series of easy-to-use tools for model visualization, polishing, simplifying, editing, and exporting. Although the subscription-based platform allows the registration of up to a thousand photos, creating substantially better models, for this paper, we tested only the free version.

The last software tested was Meshroom, an open-sourced photogrammetry software developed by AliceVision. Meshroom registers data in a process that can be followed by the user at each stage, allowing expert users to include/exclude modules, improving accuracy and finetuning of processing values. It offers no model editing tool built in the software and processes an unlimited number of pictures. For this study, we used the 2019.2 version with default settings

\section{MODELING}

Finally, model refinement was made using both proprietary software made available with restrictions (Autodesk Meshmixer) and open-sourced software (Blender).
Meshmixer is a software designed to prepare models for 3D printing. It provides tools designed specifically for mesh analysis such as slicing, overhang, stability, orientation, and query. It also offers automatic and manual mesh repair tools. In turn, Blender is an open-source modeling software widely used for 3D modeling, video FX, and animation. Similarly, it offers a wide range of tools for mesh editing, sculpting, texturing, and shading

\section{RESULTS}

\section{DATA COLLECTION}

Data collection using DSLR with no GPS tag provides good enough data for the creation of a 3D mesh model, as long as there is considerable overlap of the pictures and different vantage points, especially from various heights. Vertical vantage point, along with the horizontal vantage point variation, will allow for the triangulation of points and result in good quality models, as shown with the Art Panel.

Mesh quality was affected by the number of photos that were allowed to be processed at a time since it restricted the number of points available for comparison. The effects can be seen on the top of Figure 4, on ReCap Photos' reconstruction of the Cathedral's tower: although more pictures of the towers were available to provide more points for comparison, the software's cap of a hundred pictures affected the final result.

\section{DATA REGISTRATION}

We have compared the three software through quantitative and qualitative analysis, based on seven performance indicators. The quantitative indicators are: "cache memory required," "final file size," "triangles count in the final model," and "useful triangles count." These are 


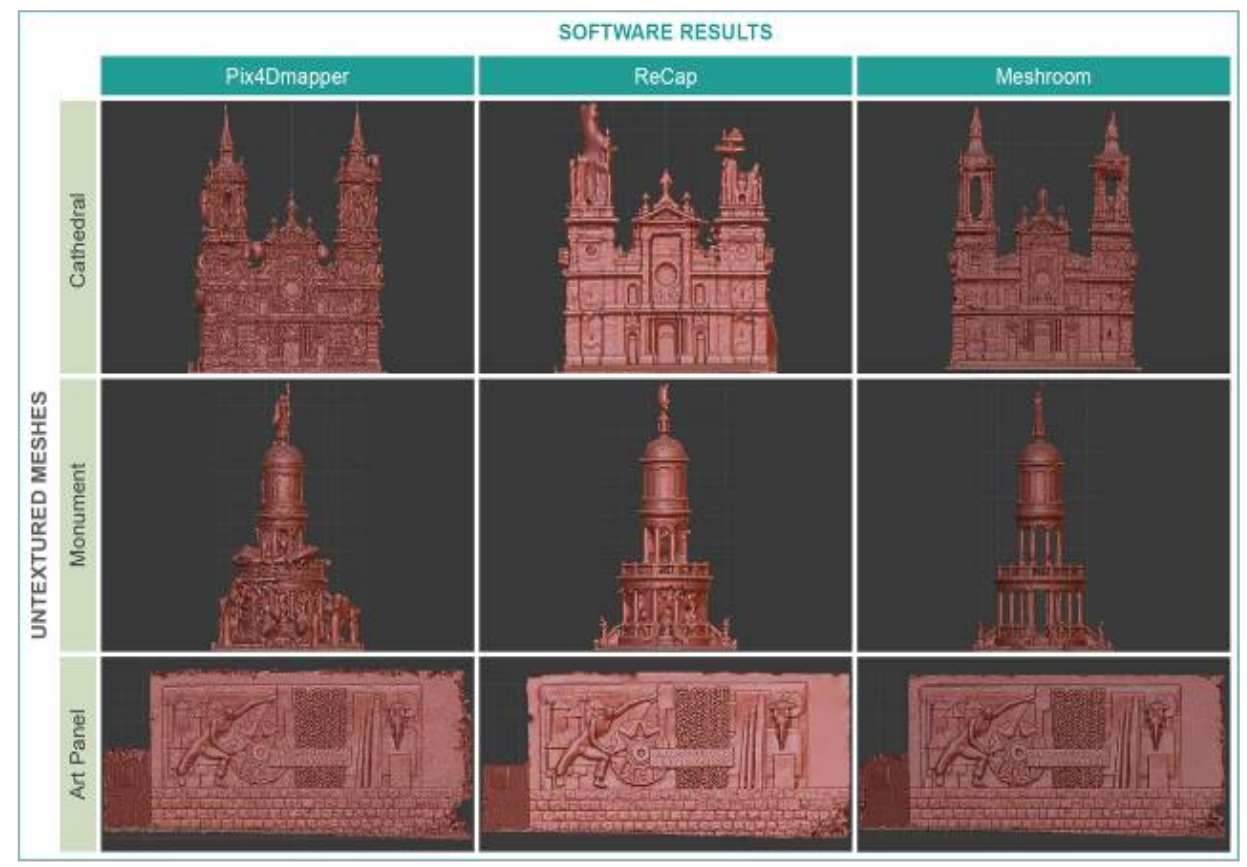

Figure 5: The three captured elements in different software without texture.

numerical data retrieved from the files folder, wavefront (OBJ) file size, triangle count of the mesh generated by each software, and triangle count of the mesh after refining the model, respectively. The qualitative indicators are "mesh sharpness," "mesh smoothness," and "detail quality," and were ranked into a four-point scale, varying from "A: excellent," "B: good," "C: average"; and "D: poor." Thus, the qualitative indicators demonstrate the authors' assessment based on the visual analysis of the models.

a) Cache memory required for data registration: while ReCap Photo used cloud processing resources, Pix4Dmapper and Meshroom use desktop processing, making the software performance conditioned to the capacities of the user's machine. Regardless, Meshroom requires much more memory than Pix4Dmapper.

b) Final file size: after registering the data, the final textured mesh generated by ReCap and Pix4Dmapper (.OBJ) are up to seven times smaller than Meshroom's files.

c) Triangles count in the final model: the number of triangles generated by each software varied significantly (Table 1). Mesh refinement approaches are different in each software: while Meshroom has an unlimited number of triangles, reaching more than 5 million in the Cathedral model, Pix4Dmapper limits (at the default setting) to 1 million, and ReCap Photo typically provides models with less than 1 million faces. In ReCap Photo, the Cathedral and Monument remained under 1 million, and the Art Panel, at 1,7 million.

d) Useful triangles count: the number of triangles roughly reduces by half when we consider only the element we intended to model, disregarding the surrounding environment and noise. The exception to this rule is the ReCap Photo, which seems to identify the element of interest better than the other software and disregard the surrounding environment, having a better ratio of useful to useless vertex (Table 1).

e) Mesh sharpness: consists of the software's ability to produce detailed meshes with sharp edges. Mesh refinement improved sharpness: while Meshroom provided models with sharper edges and finer detail, Pix4Dmapper models were faceted due to the 1 million triangles cap, and ReCap Photo generated models with smooth rather than sharp surfaces. While this may not be as perceptible in the textured model (Fig. 4), it will directly affect model measurements or 3D printing (Fig.5 and Fig. $6)$.

f) Mesh smoothness: regarding the software's ability to produce smooth flat surfaces, ReCap Photo showed the best results for the production of smooth models at any scale.

g) Detail quality: on a subjective visual evaluation of the mesh regarding the correspondence between real and modeled objects, Meshroom has offered the best detail quality for models of any scale.

Another factor that directly affected the result was the software ability to process voids: Meshroom exceeded in distinguishing the object from the background, reconstructing the Monument better than both the other software. The software's ability may be better perceived in the Cathedral's towers and the Monument's top figure, as well as the Monument as a whole. 


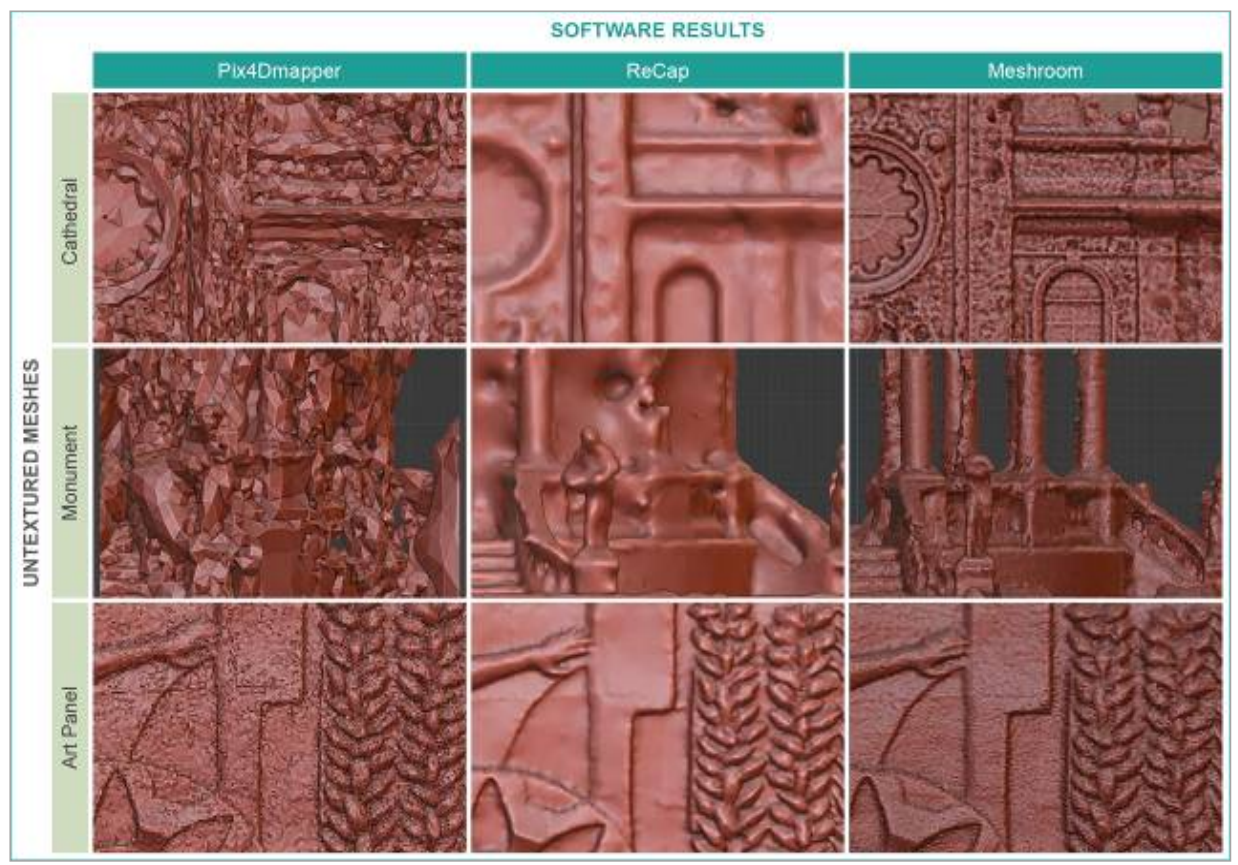

Figure 6: Details of the three captured elements in the different software without texture.

The performance indicators of each software through the registration of the three models are summarized in Table1.

Table 1: Synthesis of the registration software performances.

\begin{tabular}{|c|c|c|c|c|c|c|c|c|c|}
\hline \multirow[b]{2}{*}{ Parameter } & \multicolumn{3}{|c|}{ Cathedral } & \multicolumn{3}{|c|}{ Monument } & \multicolumn{3}{|c|}{ Art Pannel } \\
\hline & 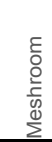 & 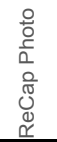 & 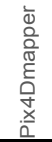 & 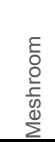 & 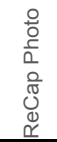 & 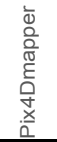 & 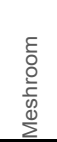 & 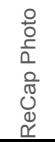 & 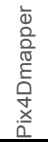 \\
\hline $\begin{array}{l}\text { Cache Required } \\
(\mathrm{Gb})\end{array}$ & 18,7 & 0,33 & 2,24 & 17,0 & 0,37 & 1,73 & 15,9 & 0,1 & 2,48 \\
\hline File size (Mb) & 380 & 55,7 & 70,4 & 326 & 69,4 & 69,7 & 281 & 184 & 68,3 \\
\hline $\begin{array}{l}\text { Triangles } \\
\text { count }\left({ }^{*} 10^{6}\right)\end{array}$ & 5,1 & 0,54 & 1,0 & 4,5 & 0,67 & 1,0 & 3,8 & 1,76 & 1,0 \\
\hline \begin{tabular}{|l} 
Useful Triangles \\
count $\left({ }^{*} 10^{6}\right)$
\end{tabular} & 4,1 & 0,38 & 0,58 & 3,8 & 0,67 & 0,47 & 3,6 & 1,2 & 0,52 \\
\hline $\begin{array}{l}\text { Mesh } \\
\text { Sharpness }\end{array}$ & A & C & B & A & C & B & A & B & B \\
\hline \begin{tabular}{|l} 
Mesh \\
Smoothness
\end{tabular} & C & A & D & C & A & D & B & A & C \\
\hline Detail Quality & $A$ & $B$ & $\mathrm{C}$ & $A$ & $B$ & C & $A$ & $B$ & $B$ \\
\hline
\end{tabular}

\section{MODELING}

The Meshmixer software is intuitive to use, and it works well for small objects that have a continuous shell, although it offers some restrictions when working with dense or complex meshes since its tools will try to process the whole mesh at once.

Blender offers a wide range of tools for mesh editing, sculpting, texturing, and shading, but it does have a steep curve of learning. It is well suited for working and editing large or dense meshes since it allows for the selection of specific faces, edges, or vertices.

Data registered with any of the three software created meshes with overlapping and complex surfaces, which was labor-intensive to cleaning with Meshmixer, especially with large files size such as those generated by Meshroom, which makes the use of tools, such as auto repair, unpractical because it too much from the computer.

Due to file size and complexity, Blender was more suitable for modeling, allowing for the selection of specific vertices for mesh editing, sculpting, and some texture repair.

\section{DISCUSSION}

For the best possible quality, data should be collected using drones. This method allows for the best vantage points of the building/façade/objects while ensuring image overlap and providing GPS tagged images. Photographs taken with a handheld camera will also provide the data required for registration, as long as they are captured by several different horizontal and vertical vantage points, with sufficient overlap. That can be done through several different methods, depending on the object: using selfiesticks, ladders, tall cars, front-facing buildings, weather balloons, cranes, amongst others.

The best quality $3 \mathrm{D}$ models were made by the opensource software Meshroom. It provided high detail, fine mesh with a high triangulation count. The models created by Meshroom had sharper detail and differentiated the object of interest from its background better than the other software. That was true, regardless of the object scale. The downside is the hardware space required for 
processing, although the process may be stopped midway and restart from the process interrupted, maintaining the progress made in the previous steps.

The main problem with the other software was the caps instituted in the default setting: Pix4Dmapper 1 million triangles cap for the mesh was suitable for smaller-scale objects but made the larger-scale objects (Monument and Cathedral) faceted and disfigured. The same problem happened with ReCap Photo: while it had the process capability to produce better models, the hundred photos cap on the free license requires a selection of photos that may compromise larger models, such as the Cathedral.

Both Pix4Dmapper and ReCap Photo had difficulties with voids in the model, especially when there are layers of structures to be modeled, as were the case of the Monument. In Pix4Dmapper, this may be overcome using the fine-tuning tools available inside the software and ReCap Photo's paid to render version that allows for up to a thousand photos may be able to process it better, but Meshroom was able to process it much better than both ReCap Photo and Pix4Dmapper with the data available and using a free license.

If the purpose of the model is only for visualization or simulation, ReCap Photo models may be the most suited since it offers light, smooth and clean meshes suitable to be experienced with texture. In contrast, Meshroom's mesh is dense and highly detailed, more suitable for close up inspection, measurements, and registry of historical buildings and heritage. For visualization with texture, Meshroom models should be decimated to create lighter models.

Modeling can be made using Blender regardless of model size, although, for smaller models, Meshmixer may be the better option since it provides a series of tools that facilitate mesh repair and the creation of a manifold model for visualization or 3D printing.

It is worth emphasizing that this analysis was made considering free to use or trial licenses, adopting the standard settings for data registration. The same software with the full license, using the best configuration, and the built-in tools available may offer significantly different results.

\section{REFERENCES}

Achille, C., Adami, A., Chiarini, S., Cremonesi, S., Fassi, F., Fregonese, L., \& Taffurelli, L. (2015). UAV-based photogrammetry and integrated technologies for architectural applications-methodological strategies for the after-quake survey of vertical structures in Mantua (Italy). Sensors (Switzerland). Retrieved from https://doi.org/10.3390/s150715520

Boeters, R., Arroyo Ohori, K., Biljecki, F., \& Zlatanova, S. (2015). Automatically enhancing CityGML LOD2 models with a corresponding indoor geometry. International Journal of Geographical Information Science, 29(12), 2248-2268. Retrieved https://doi.org/10.1080/13658816.2015.1072201

El-Hakim, S. (2001). 3D modeling of complex environments. Videometrics and Optical Methods for 3D Shape Measurement, 4309(4309), 162-173. Retrieved from http://citeseerx.ist.psu.edu/viewdoc/download?doi=10.1.1. 28.1970\&rep=rep1\&type=pdf

Gröger, G., Kolbe, T. H., Nagel, C., \& Häfele, K.-H. (2012). OpenGIS City Geography Markup Language (CityGML) Encoding Standard, Version 2.0.0. OGC Document No. 12-019, $344 . \quad$ Retrieved from https://portal.opengeospatial.org/files/?artifact_id=47842

Heipke, C., Madden, M., Li, Z., \& Dowman, I. (2016). Theme issue 'State-of-the-art in photogrammetry, remote sensing and spatial information science'. ISPRS Journal of Photogrammetry and Remote Sensing, 115, 1-2. Retrieved from https://doi.org/10.1016/j.isprsjprs.2016.03.006

Murphy, M., McGovern, E., \& Pavia, S. (2013). Historic Building Information Modelling - Adding intelligence to laser and image based surveys of European classical architecture. ISPRS Journal of Photogrammetry and Remote Sensing, 76, 89-102. Retrieved from https://doi.org/10.1016/j.isprsjprs.2012.11.006

Peña-Villasenín, S., Gil-Docampo, M., \& Ortiz-Sanz, J. (2020). Desktop vs. cloud computing software for 3D measurement of building façades: The monastery of San Martín Pinario. Measurement: Journal of the International Measurement Confederation, 149. Retrieved from https://doi.org/10.1016/j.measurement.2019.106984

Sužiedelytè-Visockienè, J., Bagdžiūnaitè, R., Malys, N., \& Maliene, V. (2015). Close-range photogrammetry enables documentation of environment-induced deformation of architectural heritage. Environmental Engineering and Management Journal. Retrieved from https://doi.org/10.30638/eemj.2015.149 